\title{
Alcoholism and Animals ${ }^{1}$
}

\author{
JAMES H. WOODS AND GAIL D. WINGER ${ }^{2}$ \\ Department of Pharmacology and Department of Psychology, \\ University of Michigan, Ann Arbor, Michigan 48104 and \\ Department of Psychiatry, Downstate Medical Center, \\ Brooklyn, New York 11200
}

\begin{abstract}
Studies in animals of alcohol-associated phenomena have begun to yield data which may provide a better understanding of human alcoholism. Four such phenomena, tolerance, dependence, hepatic damage and self-intoxication, have been demonstrated in both animals and man. The relationships among these phenomena, however, have not yet been determined in either human or nonhuman species. Tolerance to and physical dependence on alcohol have been clearly demonstrated in a number of animal species. The behavioral, physiological and biochemical correlates of tolerance and dependence are of considerable contemporary experimental interest. In particular, animal models of alcohol withdrawal signs will yield excellent preparations for the study of the management of problems associated with ethanol withdrawal in man. Chronic liver damage, a problem often associated with alcoholism, has been demonstrated in animals; however, it remains unclear whether the conditions necessary for its production are equivalent in animals and man. Through an approach based on operant conditioning, behavioral phenomena associated with ethanol-reinforced self-intoxication are being elucidated in animals and man. These findings provide a descriptive framework which could lead to a delimitation of the important variables that control ethanol intoxication in both animals and man; hence, new avenues for treatment and prevention of human alcoholism may be elaborated.
\end{abstract}

This paper is a brief discussion of some alcohol-related phenomena in animals which are of direct concern to the patient who "drinks too much" and to his physician. Our intent is to relate the progress of basic animal research to questions arising from the problems associated with human alcoholism. The amount of research on animals and alcohol is large; however, its exciting aspects are often dulled by technical jargon, and more importantly, the alcohol literature on animals is often unrelated to alcoholism. We will present only what we feel are the more. important aspects of animal research in a manner that avoids jargon and aids in a better understanding of the human disease.

The most well-known and accepted fact is that there are no "spontaneous" alcoholics among our animal brethren. This may reflect a paucity of naturallyoccurring fermented alcoholic products in their natural habitats. Certainly, when animals are given the opportunity to ingest alcohol solutions in the laboratory, they uniformly show a marked lack of interest. The general absence of significant alcohol ingestion has produced consternation anong investigators

\footnotetext{
1 Preparation of the manuscript was supported by USPHS, NIMH Grant Nos. DA 00154 and MH 16477.

" Now at Department of Psychiatry, Johns Hopkins University, Baltimore, Maryland.
}

Copyright (C) 1974 by Academic Press, Inc. 49

All rights of reproduction in any form reserved. 
searching for techniques to increase the propensity of animals to ingest alcohol. Some have reached the conclusion that such attempts may be futile. For example, Myers and Veale (38) concluded that "Alcoholism is not an affliction of the animal kingdom, but a unique human disease for which no animal analogue exists" (p. 131). We agree with a portion of this proposition, viz., that the entire gamut of phenomena of alcoholism does not and probably cannot exist in a single animal model. A survey of animal models of other human disease processes would also show marked imperfections. Nevertheless, several features of certain concomitants of alcoholism do exist in animal models; perhaps, a better tactic is to make the most of these features in attempting to better understand the etiology of these problems in human alcoholism.

The study of prognostic factors in animals has been as weak as, or weaker than, human studies (25). Hence, the agent-individual intcraction has been the focus of the vast majority of research. Of this research, our attention is directed to four alcohol-associated phenomena, each of which has been demonstrated in man and animals; each has been touted as being important to a better understanding of human alcoholism. These phenomena are alcohol tolerance, alcohol dependence, alcohol-induced hepatic damage, and ethanolreinforced self-intoxication.

\section{TOLERANCE}

Tolerance is said to have developed when, as a function of exposure to alcohol, the drug has less ability to alter behavioral and physiological function. Acute tolerance, or tolerance that develops during a single administered dose of alcohol, can be measured by relating degree of impairment to blood alcohol levels throughout the course of action of the drug. A greater degree of impairment at a given blood alcohol level during the rising phase of the blood alcohol curve, as compared to the degree of impairment at the same blood alcohol level when the blood levels are falling, has been taken as evidence of acute tolerance (35). By another criterion, a return to nearly normal function while blood levels are still high can also be considered acute tolerance (35, 40). Although the acute tolerance observed in these studies may be accounted for in part by a redistribution of alcohol away from the central nervous system to the periphery, the observations of Maynert and Klingman (28) of acute tolerance development in dogs are not subject to this interpretation. These investigators found that the blood alcohol level at which dogs recovered from alcohol-induced ataxia was as much as 230 percent greater following a high dose as compared to a low dose of alcohol. They concluded that acute tolerance developed during the course of a single, high dose of alcohol.

Chronic tolerance, or tolerance that develops over the course of several days or weeks of alcohol administration, can be measured accurately only in terms of shifts in dose-response curves. To obtain this measurement, the effect of several doses of administered alcohol must be first evaluated on a selected behavioral or physiological parameter, so that the doses of alcohol may be related to its effect. Chronic alcohol administration is then begun, at 
the end of which a second dose-effect relationship is obtained. The amount of shift of the second curve to the right of the first curve is an unambiguous indication of the degree of tolerance development in terms of the dose increment of alcohol required, following chronic administration, to produce the effects obtained by alcohol prior to chronic administration. This procedure for tolerance evaluation is both tedious and time-consuming, and it has rarely been carried out with alcohol. Investigations content with observing a change in the effectiveness of a constant daily dose of alcohol have reported a decreasing effect of alcohol in rats on such measures as seizure threshold (1), ability to stay on a tilted plane (43), and responding to avoid electric shock presentation (36). In one of the few studies in which shifts in dose-response curves were measured, tolerance to alcohol was observed in rats trained to run on a treadmill to avoid electric shock. Following 24 days of daily administration of as much as $9 \mathrm{~g} / \mathrm{kg}$ of alcohol, a 50 percent increase was observed in the amount of alcohol necessary to produce one-half the maximal response decrement (23).

The mechanisms by which tolerance develops to alcohol are largely unknown. Although the rate of alcohol metabolism is increased slightly following chronic administration (42), this cannot explain acute tolerance development where the independent measure is typically blood alcohol level. Furthermore, since increased rate of metabolism would result primarily in a decreased duration of action of parenterally-administered alcohol, it would not account for chronic tolerance observed within minutes following alcohol administration before significant amounts of alcohol have been metabolized. When a behavioral task is used to measure tolerance development, it is possible that the subjects learn to compensate for the effects of alcohol, and tolerance can be accounted for by behavioral adjustments. Le Blanc et al. (21) tested this idea by comparing tolerance development in rats given alcohol prior to behavioral tests to tolerance development in rats given alcohol following behavioral testing. The rate of tolerance development was greater in the former group, but of a magnitude equal to that of the latter group. Thus, learning was felt to modify only the rate of acquisition of tolerance. An underlying mechanism is usually considered to involve adaptation of the central nervous system to the presence of alcohol. Several hypotheses and some experimental data have focused on the potential basis of CNS tolerance to alcohol in terms of alterations in neurotransmitter release and other biochemical changes. There are discrepancies between the many theories and the small amount of data. A great deal more experimental investigation is required before obtaining any clear idea of how tolerance develops in the central nervous system (22).

The question of how tolerance might act to modify alcohol intake has not been systematically asked in experimental studies on animals or man. This is an important question since the predicted effect is that as tolerance develops, higher doses of alcohol become necessary to affect behavioral or physiological changes and more alcohol is, therefore, ingested. Although this phenomenon is generally thought to occur with narcotics, evidence that this occurs for 
alcohol is totally lacking. In animal studies where monkeys self-administered as much as $3.5 \mathrm{~g} / \mathrm{kg}$ of alcohol each day through intravenous catheters, there was no tendency for the amount of alcohol taken to increase over time (44). However, no concurrent independent measures were taken in these animals to determine whether tolerance, as reflected by shifts in dose-response curves, had developed in this situation. Thus, studies relating alcohol intake to degree of tolerance development remain to be undertaken.

Studies of tolerance development generally have produced many more questions than answers. The few carefully measured indices of degree of chronic alcohol tolerance development in animals suggest that it is not a tremendously large effect, especially when compared to narcotic tolerance (4). While increased rate of metabolism and behavioral adaptation may be responsible for some of the tolerance observed, the majority seems related to central nervous system changes which are almost completely unknown. The manner in which tolerance feeds back to alter alcohol intake in animals is unknown, largely because of problems in obtaining significant alcohol intake in animals. There is a need for careful and rigorous animal research, especially in regard to the mechanisms and significance of this phenomenon, which has been considered one of the important criteria for the diagnosis of human alcoholism (5).

\section{PHYSICAL DEPENDENCE}

Physical dependence on alcohol, like tolerance, develops during exposure to alcohol. Unlike tolerance, however, evidence for physical dependence development cannot be obtained until alcohol administration is discontinued, when the occurrence of withdrawal signs indicates that alcohol dependence has developed. These definitive withdrawal signs are usually categorized as manifestations of central nervous system hyperexcitability: tremor, tachycardia, fever, nausea, convulsions; and in man, hallucinations and delirium tremens.

A variety of techniques have been used to produce physical dependence to alcohol in animals. Since animals' voluntary consumption of alcohol is unnecessary, the drug is often administered directly via parenteral injection or gavage. This insures that alcohol gets into the organism and produces a range of dependence severity from the most mild following a single large dose of alcohol $(15,29)$ to the most severe following wceks or months of treatment (8-10). However, the dosage regimen for producing physical dependence, where relatively high and continuous blood alcohol levels are required, is difficult to maintain and investigators have often turned to other methods of alcohol administration. Excellent data on alcohol dependence in mice has been obtained using an apparatus which pumped alcohol vapor into the animals' cage. If metabolism of alcohol was blocked by injections of pyrazole, dependence to the inhaled alcohol developed in direct relation to the time of alcohol exposure and the concentration of alcohol in the air (15). Dependence to alcohol has been obtained by providing an alcohol-liquid food diet to mice as a sole source of food and fluid. Consumption of this diet by food-deprived mice resulted in large amounts of alcohol ingestion. When ethanol in the diet 
was replaced with a sucrose solution, a withdrawal syndrome ensued that consisted of hyperexcitability, tremor, and in some cases, tonic-clonic convulsions and death (14). Physical dependence to ethanol can be produced in rats using a technique where food-deprived animals receive a small food pellet every 1-2 min and drink excessive amounts of any available, relatively palatable fluid following the delivery of each pellet (11). When this fluid is a low concentration of alcohol, intoxication has resulted (24). Furthermore, as demonstrated by Falk et al. (12), when this procedure was continued on a chronic basis during which blood levels of ethanol of $150-250 \mathrm{mg} \%$ were achieved on several occasions each day over a two-month period, physical dependence in the form of tonic-clonic convulsions and death resulted within 10 hours after alcohol removal.

Other techniques have involved rhesus monkeys which self-administered alcohol intravenously in sufficient amounts to produce physical dependence. Dependence was manifested by tremors and hyperexcitability as well as by convulsions $(6,45)$.

Withdrawal reactions can be fatal in man (41), monkeys (9), and dogs (10), as well as in rats $(12)$, and mice $(14,15)$. The difference between the manner of death in man compared to that in an animal may be important in evaluating animal research in this area. In each case, if the withdrawal reaction was severe enough to cause death in animal subjects, death occurred during convulsions. Humans often evidence convulsions during alcohol withdrawal, but these are rarely fatal. Death usually occurs in humans during delirium tremens, a reaction which develops after, or in the absence of, convulsions and is occasionally accompanied by cardiovascular collapse which appears to be unresponsive to treatment (41). It may be important to determine the nature of the differences between alcohol withdrawal in humans and animals if an adequate model of alcohol dependence is to be developed for evaluation of possible treatment modalities. Attempts to resolve this discrepancy, however, will be hampered by the rather crude methods of measuring withdrawal. As pointed out by both Kalant $e t$ al. (22) and Mello (32), withdrawal signs are usually evaluated by observations of gross behavioral changes, making it difficult to quantify and compare the relative degree of dependence. Phenomena in humans such as hallucinations and perceptual disturbances are often indices of withdrawal severity, and although useful in man, they should be accompanied by reports of changes in body temperature, blood pressure, and heart rate so that comparisons can be made to animal data as it becomes available.

The valuable data which can be obtained when quantifiable measures of withdrawal are employed were exemplified in a study on the effect of various drugs on physical dependence in mice. Goldstein reported that alcohol, pentobarbital, paraldehyde, and meprobamate successfully suppressed alcohol withdrawal signs temporarily while barbital, phenobarbital, and benzodiazepines gave longer, more complete control. Phenothiazines exacerbated the withdrawal signs while chlormethiazole had no effect (16). Although the measures taken (i.e., convulsion-like signs in mice when held by their tails) have no counterpart in human withdrawal, the fact that they were 
quantifiable made possible the evaluation of the drug effects. If measures which are both quantifiable and relevant to the human condition can be developed in animals, significant data can be obtained that may be more applicable to humans.

The question of how physical dependence to alcohol might modify subsequent alcohol intake has at least been asked (32). However, it has not been satisfactorily answered, due again in part to a paucity of techniques for obtaining high amounts of voluntary alcohol ingestion in animals. It is commonly felt that the onset of withdrawal signs from narcotics is important in maintaining drug intake of this class in man and animals. It is less obvious that this is the case for alcohol. Myers et al. (37) reported that rhesus monkeys consumed no more alcohol following the production of physical dependence via administration and subsequent withdrawal of $6 \mathrm{~g} / \mathrm{kg} /$ day of alcohol. Under conditions where alcohol was self-administered by monkeys via intravenous catheters, the pattern of intake was cyclic. Several days of high alcohol intake were followed by cessation of intake and onset of withdrawal signs. Reinitiation of intake occurred when the signs had ameliorated somewhat (44). This pattern does not develop with other self-administered drugs which produce physical dependence (46), and it is possible that the onset of alcohol withdrawal led to termination rather than maintenance of self-administration. Of course, it is also possible that voluntary cessation of alcohol intake, although accompanied by withdrawal, was caused by factors unrelated to physical dependence.

Animal studies of physical dependence to alcohol have begun to provide data of relevance to the dependent human. More studies are needed in which the severity of withdrawal is related to amount and duration of alcohol administration and in which the effectiveness of various drugs in modifying withdrawal is evaluated $(15,16)$. To carry out such studies, better quantitative methods of measuring withdrawal in animals are needed. With improved measurement techniques, the relation between withdrawal in humans and withdrawal in animals could be ascertained, and the question of the effect of degree of withdrawal on subsequent alcohol intake might also be answered.

\section{LIVER PATHOLOGY}

Intake of large amounts of alcohol is often associated with alterations in liver structure and function. These alterations may be as innocuous as in an increased deposition of fat in the liver following a single dose of alcohol (7) or as severe as cirrhosis of the liver following many years of steady or periodic alcohol consumption. There has been considerable debate as to the etiology of the more chronic liver diseases which occur in association with alcoholism. While some investigators feel that alcohol is directly hepatotoxic (27), others hold strongly to the opinion that the primary role of alcohol in liver disease is to cause dietary imbalance which, in turn, is responsible for liver disease (18). There is also a question as to the progression, or lack of progression, from the fatty liver which develops in almost all individuals when they consume large amounts of alcohol, and the hepatitis and cirrhosis which develop in some 
people who consume alcohol in large quantities for many years. Although it would seem that the use of animals is the most appropriate way to answer these questions, several problems exist. First, it apparently requires several years of chronic alcohol intake before the more severe forms of liver disease develop. Such long-term animal studies are difficult and tedious and have not been particularly popular. Second, the laboratory rodent, the typical subject for these investigations, tends to develop a fatty liver under different dietary circumstances from those that produce liver damage in primates (19). Thus, the relevance of results from studies designed to determine the importance of diet as opposed to alcohol in producing fatty liver in rats is subject to question. Third, it is very difficult to develop an animal preparation that consumes alcohol in quantities that resemble those of the human alcoholic. Early evidence that alcohol consumption produced no liver damage (3) must be questioned, since under the circumstances ( $15 \%$ alcohol as the sole source of fluid to rats), very little alcohol was consumed.

More recently, investigators have produced greater alcohol intake by mixing the alcohol with a sugar solution, which is then presented as the sole source of fluid. Intake of alcohol by rats drinking alcohol-sucrose solutions has been reported in the range of $17-35 \mathrm{~g} / \mathrm{kg} / \mathrm{day}$, which is a substantial amount of alcohol (17). With a very high caloric intake from the fluid source, however, very little solid food was consumed, and protein deficiencies typically resulted. Studies using this technique have demonstrated that dietary imbalance is sufficient to cause fat deposition in the liver of rats, but they have not successfully elucidated the role of alcohol in the development of this problem.

Mixing alcohol with a high protein liquid food has helped overcome the problem of combining high alcohol intake with proper dietary balance (26). Using this technique, investigators found that rats developed a fatty liver when drinking an alcohol-liquid food diet, while rats drinking an isocaloric sucrose-liquid food or fat-liquid food diet did not develop a high level of liver triglycerides. Thus, alcohol was directly implicated in the deposition of liver fat. Other investigators have increased the protein amount in this alcohol-liquid diet and successfully prevented the fatty liver, suggesting that alcohol may serve to increase the protein requirement of the liver (18).

The question of the effect of alcohol on the liver has not been adequately answered by these studies, and due to the previously mentioned problems involved in the use of rats, and in obtaining high alcohol intake, other methods should be sought in attempting to answer this question. Preliminary reports on the effect of alcohol on the livers of dogs which received $3 \mathrm{~g} / \mathrm{kg}$ of ethanol daily via gastric intubation indicate that cirrhosis may have developed over a 34 -month period (39). The animals received a high protein liquid food, also administered via the gastric tube. This procedure may represent the most efficacious manner of obtaining adequate alcohol intake in combination with adequate dietary intake. Whenever voluntary ingestion of alcohol or food is allowed, either alcohol intake is low or food intake is low. Since these are the two confounding factors which need to be carefully controlled, it may be nec- 
essary to remove the voluntary aspect of alcohol and food consumption to ascertain the effect of each on liver structure and function.

Perhaps the most obvious conclusion to be drawn from the hundreds of research and review articles concerning alcohol-related increases in liver fat is that the critical nature of this effect is not yet understood. Nevertheless, whether ethanol is directly toxic to the liver or whether it acts indirectly by causing dietary imbalance, alcohol consumption is correlated with pathological hepatic damage in humans and animals. Critical questions concerning the progression of fatty liver to cirrhosis and the related problem of why a majority of alcoholics do not develop cirrhosis have not been systematically evaluated in animal studies. The long-term nature of these studies and the apparent finding that larger, slower growing animals are more appropriate subjects increase the difficulty involved in such evaluation.

\section{BEHAVIORAL MODELS OF ALCOHOL-REINFORCED SELF-INTOXICATION}

The pharmacologist, biochemist, and pathologist have been concerned primarily with changes that occur in animals subsequent to large amounts of alcohol intake. In each of the studies noted above on tolerance, physical dependence, and liver damage, considerable amounts of alcohol were taken by the animals; in no case were the animals given a serious option not to take alcohol. It was either administered to them directly via parenteral injection or gavage, present as vapor in the air they were breathing, mixed with the only source of food and fluid available, or present in nonaversive concentrations to animals in situations where intake of any palatable fluid is increased tremendously.

During and/or following this experience with ethanol, the propensity to ingest ethanol per se does not significantly increase; rather, the alcohol ingestion is controlled, in large part, by caloric deficit in both the case of scheduleinduced ethanol ingestion in the rat $(13,30)$ and in the case of food-deprived mice (14). Thus, the consumption of ethanol does not necessarily change the predisposition of these animals to consume additional amounts. There are, however, other ways to provide ethanol to animals that may overcome some of the difficulties associated with these approaches. It may be that the taste and smell of alcohol are sufficiently noxious that they preclude the ingestion of intoxicating amounts.

Bypassing these problems has resulted in an animal preparation that, in the absence of stress or other experimental manipulations, selects alcohol in the presence of freely available water and food, becomes profoundly and routinely intoxicated with blood alcohol levels in the $300-400 \mathrm{mg} \%$ range, and becomes physically dependent if given unlimited access to alcohol. This preparation involves rhesus monkeys with chronic intravenous catheters who press available levers and receive intravenous injections of small amounts of alcohol $(6,44,45)$.

Under these conditions, an important nexus of the agent-individual interaction occurs. The monkey, in its meanderings in the cage, happens upon the 
lever and presses it; if an alcohol injection occurs, the lever-press frequency increases over time. According to definition, alcohol is said to reinforce the response that produces it. The other consequences, e. g. gross intoxication and subsequent development of physical dependence, are not dictated by the experimental equipment but rather by the particular behavioral interaction of alcohol with the monkey. The behavioral predisposition toward ethanol is changed, and the monkey "voluntarily" consumed large amounts of ethanol without any other inducing agent. Some of the conditions affecting this relationship have begun to be described $(44,45)$, and it should not be surprising that alcohol, as an intoxicant, sets limits upon the monkey's propensity to take it. Thus, if the "bar" is open daily for limited times, the monkey initially avidly consumes; and as it becomes more inebriated, it takes less. On the other hand, if the "bar" is open around the clock and it is well-stocked, the monkey stays intoxicated virtually on a continuous basis except for periods of abstinence, much like the alcoholic who goes on binges. This whimsical description is meant to imply that we feel that alcohol's intoxicating properties are a major determinant of patterns of alcohol self-administration.

Seen from the other side of the cage, do these experiments have any other relevance to human alcoholism apart from another way of inducing physical dependence in animals? Certainly, there are now conditions under which humans and monkeys exhibit similar proclivities toward alcohol with sequalae that are part of what was once thought to be uniquely human. Likewise, if you share our predilection, it is no longer necessary to say that alcoholism is due exclusively to the need to reduce the stresses and anxieties of the human environment. In other words, the monkey preparation discussed above allows the opportunity to investigate the reinforcing property of alcohol in the absence of social sanction, work pressure, etc., and to subsequently add these factors and others to a more global evaluation. Such a conceptual program of research should be considered as being in its infancy.

Nevertheless, there is an equivalent conceptual framework for human studies that also draws upon the concept of the reinforcing property of alcohol. The experimental analysis of human alcoholic drinking may proceed as an instance of behavior maintained by subsequent ingestion of alcohol. Indeed, it was not until 1965 that such a possibility was capitalized upon by Mello and Mendelson (33) to describe the drinking patterns of human alcoholics in a research setting. Apart from their interesting findings that have been fully reviewed elsewhere (31), the point to be emphasized is that a common experimental framework exists for the study of alcohol-reinforced responding in humans and animals.

Insofar as alcohol-reinforced responding is well maintained in human alcoholics, one possibility is to simulate patterns of alcohol-reinforced drinking patterns in the research setting similar to those in natural settings. Mello and Mendelson (34) have recently suggested that such a simulation is possible. Furthermore, if alcohol-reinforced drinking patterns can be related to the requirements of its acquisition, then we might feel more confident in exploring conditions in which drinking patterns could be arranged to occur 
in a manner more compatible with other socially-sanctioned behavior, rather than to its virtual exclusion, as in some forms of human alcoholism.

This bit of fantasy is not without some experimental foundation since Hunt and Azrin (20) have recently begun to explore these possibilities within an experimental framework. They use what they call a community-reinforcement approach, which has a number of common, important elements with the descriptive approach taken by Bacon (2). It is argued by Hunt and Azrin (20) that the human alcoholic progresses toward larger and larger segments of his behavior under the control of alcohol and its pharmacological consequences, and less so under the control of the satisfactions engendered by vocational, social, recreational, and familial arrangements. They arranged that patients admitted for treatment of alcoholism state their own objectives with respect to family, vocation, etc., and then, in conjunction with the patients they set out to obtain these objectives by stepwise counseling in setting up job interviews, negotiating specifics of marital arrangements, setting up a recreational center for the clients in the program, etc. Indeed, if natural social units were not possible, a synthetic family attractive to the client was set up. If the patient drank under these arrangements, it was suggested, and in some cases explicitly arranged, that these renewed social satisfactions would be lost. For example, the wife was instructed to "discontinue physical and social contact with the client as much as possible during that time; in the extreme case she was advised to move out of the house into a motel or with a relative until the client in a sober state requested her return" $(20, \mathrm{p}$. 95). These procedures arrange that alcohol ingestion has complex, unified effects upon the drinker-he loses a lot by doing so, and all at once, rather than in a gradual fashion over the course of perhaps years. This experiment in rearranging the alcoholic's milieu was very successful: the small number of patients in treatment spent very little time drinking, in an unemployed status, away from home, or in the institution. In each case, they spent significantly less time than a matched control group that resumed drinking, their unemployed status, and time away from their families. Over the course of the six-month study, there was no tendency for the "treatment" to be less effective; the majority of clients remained completely sober.

For our purposes, Hunt and Azrin's (20) experiment suggests the plausibility of the approach with a highly successful example and, in addition, points out that massive rearrangements of natural environmental "contracts" will markedly change drinking behavior. No single experiment will convince anyone of one approach over another. However, a concentrated analysis of the behavioral characteristics of alcoholics in their environment and the change brought by rearranging it will no doubt offer a more cohesive, descriptive framework that is unified, in that problems difficult to evaluate in humans may be examined in animals within the same descriptive system. What these procedures point to is a very promising lien on the future both for rehabilitation and possible prevention of alcoholism. 


\section{REFERENCES}

1. Allan, F. D., AND SwinYard, C. A. Evaluation of tissue tolerance to ethyl alcohol by alterations in electroshock seizure thresholds in rats. Anat. Rec. 103, 419 (1949).

2. Bacon, S. D. The process of addiction to alcohol: Social aspects. Quart. J. Studies Alc. 34, $1-27$ (1973).

3. Best, C. H., HartrofT, W. S., Lucas, C. C., AND Ridout, J. H. Liver damage produced by feeding alcohol or sugar and its prevention by choline. Brit. Med.J. 2, 1001-1006 (1949).

4. Carney, J. M., Woods, J. H., And Downs, D. A. Dose-dependent production and measurement of morphine tolerance and cross tolerance. Pharmacologist 15, 236 (1973).

5. Criteria Committee, National Council on Alcoholism, Criteria for diagnosis of alcoholism. Ann. Internal Med. 77, 249-258 (1972).

6. DenEaU, G. A., YANAGita, T., AND SEevers, M. H.: Self-administration of psychoactive substances by the monkey: A measure of psychological dependence. Psychopharmacologia 16, 30-48 (1969).

7. DiLuzio, N. R. Effect of acute ethanol intoxication on liver and plasma fractions of the rat. Am. J. Physiol. 194, 453-456 (1958).

8. ElLrs, F. W., AND PICK, J. R. Ethanol-induced withdrawal reactions in the rhesus monkey. Pharmacologist 11, 256 (1969).

9. Ellis, F. W., AND PICK, J. R. Dose- and time-dependent relationships in ethanol-induced withdrawal reactions. Fed. Proc. 30, 568 (1971).

10. Essig, C. F., AND LAM, R. C. Convulsions and hallucinatory behavior following alcohol withdrawal in the dog. Arch. Neurol. 18, 626-632 (1968).

11. FALK, J. L. Analysis of water and $\mathrm{NaCl}$ solution acceptance by schedule-induced polydipsia. J. Exp. Anal. Behav. 9, 111-118 (1966).

12. FALK, J. L., SAMPSON, H. H., AND WINGER, G. Behavioral maintenance of high concentrations of blood ethanol and physical dependence in the rat. Science, 177, 811-813 (1972).

13. FreEd, E. X., AND Lester, D. Schedule-induced consumption of ethanol: Calories or chemotherapy? Physiol. Behav. 5, 555-560 (1970).

14. Freund, G. Alcohol withdrawal syndrome in mice. Arch. Neurol. 21, 315-320 (1969).

15. Goldstein, D. B. Relationship of alcohol dose to intensity of withdrawal signs in mice. $J$. Pharmacol. Exp. Therap. 180, 203-215 (1972).

16. Goldstein, D. B. An animal model for testing effects of drugs on alcohol withdrawal reactions. J. Pharmacol. Exp. Therap. 183, 14-22 (1972).

17. Gomez-Dumm, C. L. A., Porta, E. A., Hartroft, W. S., And Koch, O. R. New experimental approach in study of chronic alcoholism. II. Effects of high alcohol intake in rats fed diets of various adequacies, Lab. Investigation 18, 365-378 (1968).

18. Hartroft, W. S., And Ponta, E. A. Pathogenesis of fatty liver and cirrhosis: Histopathological approach, in "Alcohol and Alcoholism" (R. E. Popham, Ed.) p. 212-222, University of Toronto Press, Toronto, 1969.

19. HoffBauer, F. W. AND ZaKi, F. Choline deficiency in baboon and rat compared. Arch. Path. 79, 364-369 (1965).

20. HUN', G. M., AND AZRIN, N. H. A community-reinforcement approach to alcoholism. Behav. Res. Therapy 11, 91-104 (1973).

21. KaLANT, H., LEBLANC, A. E., AND GiBBins, R. I. Tolerance to and dependence on ethanol, in "Biological Basis of Alcoholism" (Y. Israel and J. Mardones, Eds.) pp. 235-269, Wiley Interscience, New York, 1971.

22. LeBlanc, A. E., Kalanx, H., Gibbins, R. J., and Berman, N. D. Acquisition and loss of tolerance to ethanol by the rat. J. Pharmacal. Exp. Therap. 168, 244-250 (1969).

23. LeBlanc, A. E., Gibbins, R. J., AND Kalant, H. Behavioral augmentation of tolerance to ethanol in the rat. Psychopharmacologia 30, 117-122 (1973).

24. LESTER, D. Self-maintenance of intoxication in the rat. Quart. J. Studies Alc. 22, 223-231 (1961). 
25. LESTER, D. Self-selection of alcohol by animals, human variation and etiology of alcoholism. Quart.J. Studies Alc. 27, 395-438 (1966).

26. Lieber, C. S., Jones, D. P., Mendelson, J., And DeCarti, L. M. Fatty liver, hyperlipemia, and hyperuricemia produced by prolonged alcohol consumption, despite adequate dietary intake. Trans. Assoc. Am. Physicians 1, 289-301 (1963).

27. LieBer, C. S., AND RuBin, E. Ethanol-A hepatotoxic drug. Gastroenterology 54, 642-646 (1968).

28. MaYNERT, E. W., AND Klingman, G. I. Acute tolerance to intravenous anesthetics in dogs. J. Pharmacol. Exp. Therap. 128, 192-200 (1960).

29. MCQUarite, D. G., AND Fingl, E. Effects of single doses and chronic administration of ethanol on experimental seizures in mice. J. Pharmacol. Exp. Therap. 124, 264-271 (1958).

30. MEisch, R. A., AND ThOmpson, T. Ethanol as a reinforcer: effects of fixed-ratio size and food deprivation. Psychopharmacologia 28, 171-183 (1973).

31. Mello, N. K. Behavioral studies of alcoholism, in "The Biology of Alcoholism" (B. Kissin and H. Begleiter, Eds.), Vol. 2, pp. 219-291, Plenum Press, New York, 1972.

32. MeLLo, N. K. A review of methods to induce alcohol addiction in animals. Pharmacol. Biochem. Behav. 1, 89-101 (1973).

33. Mello, N. K., ANd Mendelson, J. H. Operant analysis of drinking patterns of chronic alcoholics. Nature 206, 43-46 (1965).

34. Mello, N. K., AND MEndelson, J. H. Drinking patterns during work-contingent and noncontingent alcohol acquisition. Psychosom. Med. 34, 139-164 (1972).

35. Mirsky, I. A., Piker, P., Rosenbaum, M., and Lederer, H. "Adaptation" of the central nervous system to varying concentrations of alcohol in the blood. Quart. J. Studies Alc. 2, $35-45$ (1941).

36. MoskowitZ, H., AND WAPNER, M. Studies on the acquisition of behavioral tolerance to alcohol. Quart. J. Studies Alc. 25, 619-626 (1964).

37. Myers, R. D., Stoltman, W. P., AND Martin, G. E. Effects of ethanol dependence induced artificially in the rhesus monkey on the subsequent preference for ethyl alcohol. Physiol. Behav. 9, 43-48 (1972).

38. MYERS, R. D., AND VEALE, W. L. The determinants of alcohol preference in animals, in "The Biology of Alcoholism" (B. Kissin and H. Begleiter, Eds.), Vol. 2, pp. 131-168, Plenum Press, New York, 1972.

39. PICK, J. R. AND Ellis, F. W. Some histopathologic findings in a canine model of ethanol dependence. Pharmacologist 15, 159 (1973).

40. Story, J. L., EidelberG, E., AND French, J. D. Electrographic changes induced in cats by ethanol intoxication. Arch. Neurol. 5, 565-570 (1961).

41. Victor, M., AND Adams, R. D. The effect of alcohol on the nervous system. Res. Publ. Assoc. Nerv. Ment. Disorders 32, 526-573 (1953).

42. VIDELA, L., AND ISRAEL, Y. Factors that modify the metabolism of ethanol in rat liver and adaptive changes produced by chronic administration. Biochem. J. 118, 275-281 (1970).

43. Wallgren, J., AND LINDBoHM, R. Adaptation to ethanol in rats with special reference to brain tissue respiration. Biochem. Pharmacol. 8, 423-424 (1961).

44. Winger, G., AND WOODS, J. H. The reinforcing property of ethanol in the rhesus monkey. I. Initiation, maintenance and termination of intravenous ethanol-reinforced responding. Ann. N.Y. Acad. Sci. 215, 162-175 (1973).

45. WoOdS, J. H., IKOMI, F., AND Winger, G. The reinforcing property of ethanol, in "Biological Aspects of Alcohol" (M. K. Roach, W. M. McIsaac, and P. J. Creaven, Eds.) p. 371, University of Texas Press, Austin, Tex., 1971.

46. WoOds, J. H., AND Schuster, C. R. Regulation of drug self-administration, in "Drug Dependence" (R. T. Harris, W. M. McIsaac, and C. R. Schuster, Eds.), p. 158, University of Texas Press, Austm, Tex., 1970. 